\title{
Robust indirect adaptive control of the electrohydraulic velocity control systems
}

\author{
W.-S.Yu, PhD
}

T.-S.Kuo, PhD

Indexing terms: Indirect adaptive control, Estimation, Robust control, Computer applications

\begin{abstract}
A robust version of an indirect adaptive control scheme with self-excitation capability to the problem of controlling velocity of the electrohydraulic servosystems subject to unmodelled dynamics and load disturbances is proposed. The scheme contains an effective gradient least squares estimator using a dead zone technique and a certainty equivalence based adaptive controller. A design procedure for synthesising the adaptive controller is formulated by using the pole-placement technique, where a signal is created and fed back to the control law such that it has self-excitation capability and thus the stability of the closed-loop control system can be achieved. A series of simulations are performed to demonstrate the effectiveness of the proposed scheme. The results show that the proposed scheme is fairly robust to the control systems with uncertainties as well as improved performance characteristics, compared with that of the suboptimal PID control scheme with constant feedback gain and that of the adaptive model following control scheme.
\end{abstract}

\section{List of symbols}

$K=$ valve constant, $\mathrm{m}^{3} / \mathrm{sec} /\left(\mathrm{mA}\left[\left[\mathrm{N} / \mathrm{m}^{2}\right]\right)\right.$
$Q_{l}=$ load flow rate of servovalve, $\mathrm{m}^{3} / \mathrm{s}$
$P_{s}=$ supply pressure, $\mathrm{N} / \mathrm{m}$
$P_{l}=$ load pressure across cylinder, $\mathrm{N} / \mathrm{m}^{2}$
$m=$ total mass of piston and load, $\mathrm{Ns} \mathrm{s}^{2} / \mathrm{rad}$
$C_{v}=$ viscous damping coefficient, $\mathrm{Ns} / \mathrm{m}$
$F_{l}=$ external load disturbance, $\mathrm{N}$
$C_{a}=$ piston ram area, $\mathrm{m}^{3} / \mathrm{rad}$
$C_{l}=$ total leakage coefficient, $\mathrm{m}^{5} /(\mathrm{Ns})$
$V_{t}=$ total volume of valve and $\mathrm{cylinder}$ chamber, $\mathrm{m}^{3}$
$C_{0}=$ bulk modulus of the oil, $\mathrm{N} / \mathrm{m}^{2}$

(C) IEE, 1996

IEE Proccedings online no. 19960519

Paper first received 26th September 1995 and in revised form 11th March 1996

W.-S. Yu is with the Department of Electrical Engineering. Tatung Institute of Technology, 40 Chung-Shan North Rd. 3rd Sec., Taipei, Taiwan 10451, Republic of China

T.-S. Kuo is with the Department of Electrical Engineering, National Taiwan University, 1 Roosevelt Rd. Sec. 4, Taipei, Taiwan, Republic of China

$$
\begin{aligned}
& C_{d}=\text { gain of nonlinear load disturbance } \\
& \dot{\psi}=\text { velocity of the piston, } \mathrm{m} / \mathrm{s} \\
& u=\text { input current of servovalve, } \mathrm{mA}
\end{aligned}
$$

\section{Introduction}

Electrohydraulic servosystems are widely used in control system applications for position or velocity controls such as mobile, airborne, and stationary equipment which require servos to convert electrical controls to large mechanical propulsion forces $[1-3,5,6]$. The simulated system in this paper is shown in Fig. 1. It consists essentially of a power cylinder and a servovalve that controls oil flow. Owing to the flow pressure properties and to the load system motion itself, the dynamic characteristic between the power cylinder and servovalve is often complex and highly nonlinear, which will result in the time-varying of the pressure gain and flow gain obtained from the linearised hydraulic system for a specified operating point.

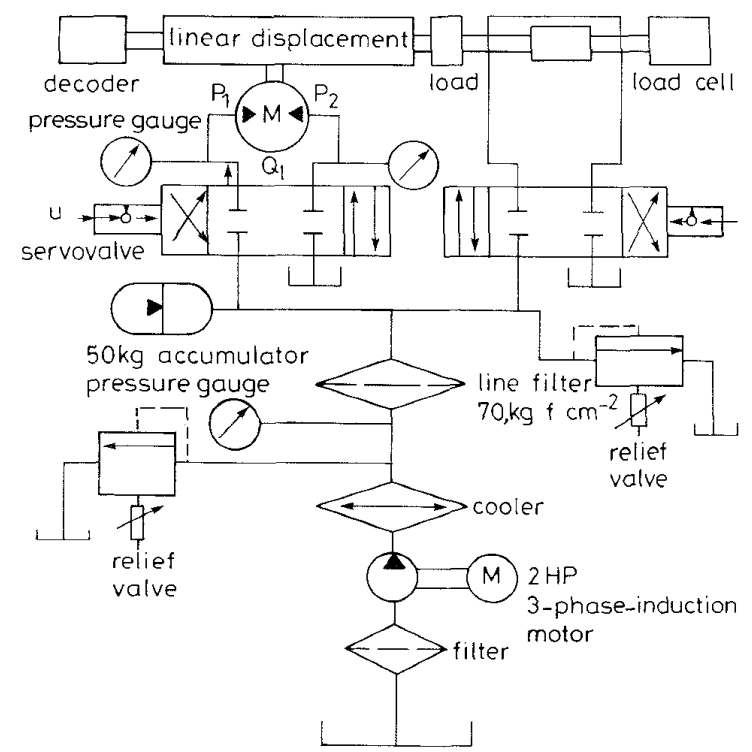

Fig. 1 Schematic diagram of electrohydraulic velocity control system

Therefore, a linear model used for describing a hydraulic system should include unmodelled dynamics. In $[2,3]$, the authors proposed a model reference adaptive control scheme for the problem of controlling the position of the system using the linearisation technique for a fixed constant, nominal operating point and considering no external load disturbances. As for the velocity 
control problem, the authors [5] presented an adaptive model following control scheme to tackle the system with changing operating points and linear load disturbances. However, since the load disturbances of the hydraulic system depend on the velocity of the piston, and are always nonlinear and time-varying, it is difficult to consider the stability of the system only with linear load disturbances or with any fixed operating point. Furthermore, it is hard to exhibit good efficiency and performances by using conventional control schemes for the nonlinear hydraulic systems.

In recent years, numerous approaches have been made in the literature towards achieving global stability and convergence of the control system using the indirect adaptive control scheme without external persistently probing signals [7, 9-13]. In this control scheme, the basic idea is to constitute an on-line estimation algorithm to estimate plant model parameters and then to obtain a stabilising controller based on the certainty equivalence principle such that the stability of the closedloop system can be achieved. In general, the external load disturbance always varies in an unknown fashion, although it is bounded, and the unmodeled dynamics exist due to the fact that the chosen plant model does not give complete descriptions of the controlled plant. So, the estimated model from an on-line estimation can never capture the true system model, which would push the estimation into poor excitation. It is therefore better to obtain an on-line linear model with unmodelled dynamics for use as the basis of control system design by dead-zone technique to avoid the phenomenon of poor excitation $[11,15,16,18,24]$.

In this paper, we propose a certainty equivalence based indirect adaptive contrel (IAC) scheme with selfexcitation capability using the pole-placement technique for the problem of controlling the velocity of the hydraulic system subject to unmodelled dynamics and load disturbances. The control scheme is an outgrowth of the work of $[16,18,24]$, which can be used to guarantee global stability of the controlled system without being corrupted by the uncertainties. To estimate the parameters of the plant model, we use the gradient least squares dead-zone estimator $[16,18]$ to obtain the best curve fitting data on-line. It has been shown that this estimator has monotonic parameter error reduction capability with an easily added norm-bound dead-zone errors given by the plant output and control input histories and uncertainties. Hence, it can treat a nonconservative uncertain signal energy bound and give a better transient performance of the system. In addition, it is computationally efficient and only requires approximately the same memory and computation time as recursive least squares. It is shown that, based on such an effective estimator, a design procedure for synthesising the certainty equivalence based adaptive controller is formulated by using the pole-placement technique, wherein a signal is created and fed back to the control law such that the control scheme has selfexcitation capability. Thus, when it is applied to control the velocity of the hydraulic servosystem, the stability of the closed-loop control system can be achieved without being corrupted by the nonlinearity of the flow pressure property, the variations of operating points of the hydraulic system, and the load disturbances. A series of simulations are performed to demonstrate the effectiveness of the proposed scheme. The results show that the proposed scheme is fairly robust to the systems with uncertainties and also improves performance characteristics, as compared with that of the suboptimal PID control scheme with constant feedback gain [4] and that of the adaptive model following control scheme [5].

\section{Derivation of the indirect adaptive control algorithm}

\subsection{Modelling of the plant}

Consider an uncertain plant $P(p)$ which is described in a stable factor form by

$$
\begin{gathered}
y(t)=\frac{\left(B^{\#}(p)+\Delta B(p)\right) / \Lambda(p)}{\left(A^{\#}(p)+\Delta A(p)\right) / \Lambda(p)} u(t)=\frac{B_{u}(p)}{A_{u}(p)} u(t) \\
A^{\#}(p)+\Delta A(p)=p^{n}+\left(A_{n-1}^{\#}+\Delta A_{n-1}\right) p^{n-1}+\cdots+\left(A_{0}^{\#}+\Delta A_{0}\right) \\
B^{\#}(p)+\Delta B(p)=\left(B_{m-1}^{\#}+\Delta B_{m-1}\right) p^{m-1}+\cdots \\
\quad+\left(B_{0}^{\#}+\Delta B_{0}\right)(m \leq n) \\
\Theta^{\mathrm{T}}=\left[A_{n-1}^{\#} \cdots A_{0}^{\#} B_{m-1}^{\#} \cdots B_{0}^{\#}\right] \\
\Delta \Theta^{\mathrm{T}}=\left[\Delta A_{n-1} \cdots \Delta A_{0} \Delta B_{m-1} \cdots \Delta B_{0}\right]
\end{gathered}
$$

where $p \triangleq d / d t, u(t)$ and $y(t)$ are the command input and plant output, respectively, and $\Lambda(p)=\left(p+\lambda_{1}\right) \ldots$ $\left(p+\lambda_{n}\right)$ is a known, strictly Hurwitz polynomial. Moreover, $A^{\#}(p)$ and $B^{\#}(p)$ are the nominal part of the plant, and $\triangle A(p)$ and $\triangle B(p)$ are the signals which arise because of time variation of system parameters and external load disturbance. The following a priori information will be assumed about the process:

(A1) $A^{\#}(p)$ and $B^{\#}(p)$ are coprime.

(A2) $\|\Delta \Theta\|<\delta, \forall t$ where $\|\cdot\|$ denotes the usual Euclidean norm on $\mathbf{R}^{n+m}$ and $\delta$ is a some, known positive constant.

(A3) $n$ and $m$ are known.

The uncertain plant described above can be reformulated as

$$
A^{\#}(p) y_{f}(t)=B^{\#}(p) u_{f}(t)+w_{f}(t)
$$

where $u_{f}(t)$ and $y_{f}(t)$ are the filtered signals of the command input $u(t)$ and plant output $y(t)$, respectively, satisfying

and

$$
\begin{aligned}
& \Lambda(p) u_{f}(t)=u(t) \\
& \Lambda(p) y_{f}(y)=y(t)
\end{aligned}
$$

$$
\begin{gathered}
w_{f}(t)=-\Delta \Theta^{\mathrm{T}} \phi(t)=p^{n} y_{f}(t)+\Theta^{\mathrm{T}} \phi(t) \\
\phi^{\mathrm{T}}(t)=\left[p^{n-1} y_{f}(t) \cdots y_{f}(t)-p^{m-1} u_{f}(t) \cdots-u_{f}(t)\right]
\end{gathered}
$$

\subsection{Adaptive control algorithm}

In the adaptive control system, since $\Theta$ is unknown and only the plant input and output are measurable, we are in a position to use the gradient least squares deadzone estimator to estimate the parameters of the plant model from the plant input and output. Let $\theta(t)$ be an estimate of $\Theta$. Since $\phi(t)$ is constructible in real time and the parameter estimate $\theta(t)$ is known at time $t$, $w_{f}(t)$ defined in eqn. 9 for each fixed $t$ is estimated according to the following:

$$
\begin{aligned}
\hat{w}_{f}(t, \tau) & =p^{n} y_{f}(\tau)+\theta^{\mathrm{T}}(\tau) \phi(\tau) \\
& =\theta^{\mathrm{T}}(\tau) \phi(\tau)+w_{f}(\tau) \quad 0 \leq \tau \leq t
\end{aligned}
$$


where $\tilde{\theta}(t)=\theta(t)-\Theta$. Let the identification error be defined by

$$
e(t)=p^{n} y_{f}(t)-\theta^{\mathrm{T}}(t) \phi(t)=w_{f}(t)+\tilde{\theta}^{\mathrm{T}}(t) \phi(t)
$$

Since the difference $\theta(t)-\theta(\tau), \tau \in[0, t]$, is known to the estimator, one can thus construct the functional correlation error signal

$$
\begin{aligned}
\eta(t, \tau) & =\hat{w}_{f}(t, \tau)+(\theta(t)-\theta(\tau))^{\mathrm{T}} \phi(\tau) \\
& =\tilde{\theta}^{\mathrm{T}}(t) \phi(\tau)+w_{f}(\tau) \quad 0 \leq \tau \leq t(\text { eqn. } 12)
\end{aligned}
$$

To construct an estimation algorithm, it is assumed that $w_{f}(t)$ and its derivatives satisfy

$$
\|w(t)\| \leq \mu d(t) \quad \forall t
$$

where $w(t)=\left[\begin{array}{lllll}0 & \ldots & 0 & p^{n-1} w_{f}(t) \ldots w_{f}(t)\end{array}\right]^{T}, \mu$ is some positive scalar and $d(t)$ is defined by

$p d(t)=-\beta_{1} d(t)+\beta_{2}\left(\left|u_{f}(t)\right|+\left|y_{f}(t)\right|+1\right) \quad d(0)>-\frac{\beta_{2}}{\beta_{1}}$

where $\beta_{1}, \beta_{2}>0$ and $\beta_{1}+\beta_{3}<\min \left(\lambda_{1}, \ldots, \lambda_{n}\right)$ for some $\beta_{3}>0$.

The gradient least squares algorithm for identifying the parameters of the plant model is given by where

$$
p \theta(t)=p \tilde{\theta}(t)=-\gamma(t) I(t) q(t) \quad \theta(0)
$$

$$
\begin{aligned}
q(t) & =\int_{0}^{t} e^{-c_{1}(t-\tau)} \phi(\tau) \eta(t, \tau) d \tau \quad c_{1}>0 \\
\gamma(t) & >0, \quad \forall t \\
I(t) & = \begin{cases}1 & \text { if }\|\eta(t)\|_{c_{1}}>\mu \bar{d}(t) \\
0 & \text { otherwise }\end{cases} \\
\|\eta(t)\|_{c_{1}}^{2} & =\int_{0}^{t} e^{-c_{1}(t-\tau)} \eta^{2}(t, \tau) d \tau \\
\bar{d}(t) & \triangleq \sup _{0 \leq \tau \leq t}|d(\tau)|
\end{aligned}
$$

Proposition 1: [16-18] The estimation algorithm defined by eqn. 18 has the following properties:

(i) Letting $\zeta(t)=\operatorname{dist}(\theta(t), \Theta)$ where $\operatorname{dist}(\cdot, \cdot)$ is a given distance, then

$$
p\left(\zeta^{2}(t)\right) \leq-2 \gamma(t) I(t)\left(\|\eta(t)\|_{c_{1}}-\mu \bar{d}(t)\right)\|\eta(t)\|_{c_{1}} \leq 0 \forall t
$$

with equality if and only if $p \theta(t)=0$.

(ii) 'Transient performance:'

$$
\int_{0}^{\infty} 2 \gamma(t) I(t)\left[\|\eta(t)\|_{c_{1}}-\mu \bar{d}(t)\right]^{+}\|\eta(t)\|_{c_{1}} d t \leq \zeta^{2}(0)
$$

where $[\cdot]^{+}$denotes the positive part

From eqn. 18, we can make up the estimated polynomials $\hat{A}(\theta(t), p)$ and $\hat{B}(\theta(t), p)$ for the controlled plant. Let $\bar{S}(\Theta)$ and $\bar{S}(\theta(t))$ be the Sylvester matrices constituted by the coefficients of polynomials $\left(A^{\#}(p), B^{\#}(p)\right)$ and $\hat{A}(\theta(t), p), \hat{B}(\theta(t), p))$, respectively. By the coprimeness of $A^{\#}(p)$ and $B^{\#}(p)$, there exist two unique polynomials

$$
\begin{aligned}
& R(p)=p^{n}+R_{n-1} p^{n-1}+\cdots+R_{1} p+R_{0} \\
& S(p)=S_{n-1} p^{n-1}+\cdots+S_{1} p+S_{0}
\end{aligned}
$$

such that

$$
R(p) A^{\#}(p)+S(p) B^{\#}(p)=\Xi(p)
$$

where $\Xi(p)=p^{2} n+\xi_{2 n-1} p^{2 n-1}+\ldots+\xi_{0}$ is a desired closed-loop characteristic polynomial. Following the assumptions A1-A3 in Section 2.1, the certainty equivalence based robust adaptive controller is given by

$R\left(\theta_{a}(t), p\right) u_{f}=T(p) r_{f}(t)-S\left(\theta_{a}(t), p\right) y_{f}(t)+n_{0}(t)(27)$

where $r_{f}(t)=r(t) / \Lambda(p)$ is an external reference input and $T(p)=T_{n-1} p^{n-1}+\ldots+T_{1} p+T_{0}$ which can be selected properly to achieve tracking purpose. Moreover, $R\left(\theta_{a}, p\right)$ and $S\left(\theta_{a}, p\right)$ are evaluated from the following: $R\left(\theta_{a}(t), p\right) \hat{A}\left(\theta_{a}(t), p\right)+S\left(\theta_{a}(t), p\right) \hat{B}\left(\theta_{a}(t), p\right)=\Xi(p)$

and $\theta_{a}(t)$ and $n_{0}(t)$ are evaluated as follows:

$$
\text { If }|\operatorname{det}(\bar{S}(\theta(t)))| \leq \rho>0 \text {, then }
$$$$
\theta_{a}(t)=\theta(t) \text { with } \bar{t}=\max \{\underline{t} \underline{t}<t \text { and }|\operatorname{det}(S(\theta(\underline{t})))|>\rho\}
$$

$$
\left.\begin{array}{rl}
n_{0}(t) & =n_{1}(t)+n_{2}(t) \\
n_{1}(t) & =n \alpha \max _{i}\left|T_{i}\right| \bar{r}, \quad 0<\alpha<\infty \\
n_{2}(t) & =\kappa \mu \bar{d}(t), \quad \kappa>0 \\
\bar{r} & =\sup _{\ell}\left\{p^{n} r_{f}(t) p^{n-1} r_{f}(t) \cdots r_{f}(t)\right\}
\end{array}\right\}
$$

Else

$$
\begin{aligned}
& \theta_{a}(t)=\theta(t) \\
& n_{0}(t)=0
\end{aligned}
$$

Note that the initial value $\theta(0)$ must satisfy $|\operatorname{det}(\bar{S}(\theta(0)))|>\rho$. It is seen that the adaptive control scheme takes the form of an adaptively tuned linear controller with an additional feedback signal $n_{0}(t)$. Also, the reference signal is compensated by the signal $n_{1}(t)$, the control parameters variations are completely removed when $|\operatorname{det}(\bar{S}(\theta(0)))| \leq \rho$, and the effect of the uncertainties are cancelled by $n_{2}(t)$. The latter produces also the required amount of excitation.

Eqn. 27 can be written as

$$
\begin{aligned}
p^{n} u_{f}(t)= & -R_{n-1}\left(\theta_{a}(t)\right) p^{n-1} u_{f}(t)-\cdots-R_{0}\left(\theta_{a}(t)\right) u_{f}(t) \\
& +T_{n-1} p^{n-1} r_{f}(t)+\cdots+T_{0} r_{f}(t) \\
& -S_{n-1}\left(\theta_{a}(t)\right) p^{n-1} y_{f}(t)-\cdots-S_{0}\left(\theta_{a}(t)\right) y_{f}(t) \\
& +n_{0}(t)
\end{aligned}
$$

Then the control law eqn. 33 provides $p^{n} u_{f}(t)$ which together with eqn. 7 allows the computation of the actuator input $u(t)$ as follows:

$$
u(t)=p^{n} u_{f}(t)+\lambda_{n-1} p^{n-1} u_{f}(t)+\cdots+\lambda_{0} u_{f}(t)
$$

Given the signals $y(t)$ and $u(t)$, the filtered signals $y_{f}(t), u_{f}(t)$ and their differentiations, and the estimate $\theta(t)$, the purpose of this paper is to design a certainty equivalence based indirect adaptive control algorithm eqns. 18 and 27 such that the input signal $u(t)$ and output signal $y(t)$, and the estimated parameter errors will be uniformly bounded subject to the unmodelled dynamics and load disturbances.

Proposition 2: There exists a positive scale $k_{1}$ and any unit vector $\mathbf{v}$ such that $\left|\mathbf{v}^{T} \phi(t)\right| /(\mu \bar{d}(t)) \geq k_{1} \kappa-\rho(t)$ where $\rho(t)$ is bounded for all $t$. The proof is shown in Appendix 7.1.

Proposition 3: The estimated parameter error is uniformly bounded for all $t$. The proof follows directly [11].

We can now establish the stability of the adaptive control system. 
Theorem 1 : With the uncertain dynamics satisfying eqn. 16, when the adaptive control algorithm eqns. 18 and 27 is applied to the system eqn. 1, it ensures that $u(t)$ and $y(t)$ are uniformly bounded for all $t$.

The proof is very similar to that of Appendix $F$ in [24] and is, therefore, omitted.

From the above analysis, the indirect adaptive controller design procedure is delineated as follows:

Step 1: Select the plant model with proper dimension to construct $\theta(t)$ and $\phi(t)$, and a proper filter $1 / \Lambda(p)$.

- Select a stability margin $\rho$ and $\theta(0)$ such that $\mid \operatorname{det}(\bar{S}$ $(\theta(0))) \mid>\rho$.

- Select the characteristic polynomial $\Xi(p)$.

- Give the values for $\phi(0), c_{1}, \beta_{1}, \beta_{2}, \alpha, \kappa, \mu, d(0)$ and $\gamma(t)$.

Step 2: Update $\hat{A}_{j}, j=0,1, \ldots, n-1$, and $\hat{B}_{j}, j=0,1, \ldots$, $m-1$, using the update law in eqn. 18.

Step 3: Calculate $R\left(\theta_{a}(t), p\right)$ and $S\left(\theta_{a}(t), p\right)$ from eqn. 26 and select $T^{\prime}(p)$ properly to achieve tracking purpose.

Step 4: Then the adaptive control law is attained from eqn. 27.

The Steps 2-4 are repeated at each sample time.

Remarks: In Step 3, if $\left|R_{0}(\theta \mathrm{a}(t))\right| \leq \epsilon$ for a small positive value $\epsilon$, one can freeze $u_{f}(t)$ to keep safe from a very large control input since the freezing technique [21] is still applied.

If the output performance is unsatisfactory, then we can increase the value $\mu$ to cope with the gain of the signal corruption and repeat Steps 2-4.

From proposition 2 and theorem 1, if the identifier or the control law grows without bound and/or $u(t)$ and $y(t)$ blow up, then modify the values $\alpha$ and $\kappa$ from eqn. 30, and repeat Steps 2-4.

\section{Simulations and discussions}

\subsection{Simulations}

A mathematical model for the electrohydraulic velocity control system was derived in Appendix 7.2. The parameters of the hydraulic servosystem used for simulations are listed in Table 1.

Table 1: Parameters of electrohydraulic servosystem

\begin{tabular}{lll}
\hline Parameter & Value & Dimension \\
\hline$C_{a}$ & $1.32 \times 10^{-5}$ & $\mathrm{~m}^{3} / \mathrm{rad}$ \\
$C_{v}$ & 88.2 & $\mathrm{~N} . \mathrm{s} / \mathrm{m}$ \\
$C_{t}$ & $2.24 \times 10^{-10}$ & $\mathrm{~m}^{5} /(\mathrm{N} . \mathrm{s})$ \\
$K$ & $1.62 \times 10^{-4}$ & $\mathrm{~m}^{3} / \mathrm{s} /\left(\mathrm{mA} \times \mathrm{V}\left(\mathrm{N} / \mathrm{m}^{2}\right)\right.$ \\
$u_{\max }$ & 15 & $\mathrm{~mA}$ \\
$u_{*}$ & 0.015 & $\mathrm{~mA}$ \\
$m$ & 0.534 & $\mathrm{~N} \times \mathrm{s}^{2} / \mathrm{rad}$ \\
$P_{s}$ & $6.86 \times 10^{5}$ & $\mathrm{~N} / \mathrm{m}^{2}$ \\
$V_{t}$ & $1.79 \times 10^{-3}$ & $\mathrm{~m}^{3}$ \\
$C_{0}$ & $3.43 \times 10^{7}$ & $\mathrm{~N} / \mathrm{m}^{2}$ \\
$n$ & 2 & \\
\hline
\end{tabular}

From Table 1, the minimum and maximum values of $K_{q}$ and $K_{c}$ in eqn. 51 can be given by

$$
\begin{aligned}
& 0.07747 \leq K_{q} \leq 0.15493 \\
& 1.27 \times 10^{-9} \leq K_{c} \leq 2.54 \times 10^{-9}
\end{aligned}
$$

and thus the parameters of the mathematical model of the electrohydraulic velocity control system in eqn. 54 are

$$
\ddot{y}(t)+A_{1} \dot{y}(t)+A_{0} y(t)=B_{0} u(t)+w(t)
$$

where $A_{0}=174.6736, A_{1}=166.2256,492.032 \leq B_{0}$ $\leq 984$ and $w(t)=1.97948 C_{d} y^{2}(t)-3.7453 C_{d} y(t) \dot{y}(t)$.

(i) Step 1: A parametric model of the following form was fitted to the on-line data to the system:

$$
\frac{\hat{B}\left(\theta_{a}(t), p\right) / \Lambda(p)}{\hat{A}\left(\theta_{a}(t), p\right) / \Lambda(p)}=\frac{\hat{B}_{0} / \Lambda(p)}{\left(p^{2}+\hat{A}_{1} p+\hat{A}_{0}\right) / \Lambda(p)}
$$

that is, $\theta(t)=\left[\begin{array}{lll}\hat{A}_{1} & \hat{A}_{0} & \hat{B}_{0}\end{array}\right]^{T}$ and $\phi(t)=\left[p y_{f}(t) y_{f}(t)-u_{f}(t)\right]^{T}$. Let the filter $1 / \Lambda(p)$ having a roll-off at roughly $20 \mathrm{rad} / \mathrm{s}$ be given by

$$
\frac{1}{\Lambda(p)}=\frac{1}{p^{2}+50 p+625}
$$

The position of the filter in the simulated system is shown in Fig. 2.

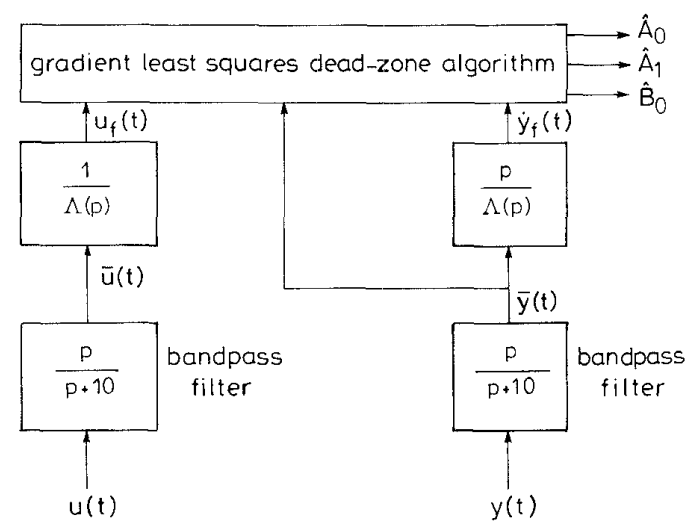

Fig.2 Filters for paramter estimation algorithm

Letting $\rho=2.25 \times 10^{-4}$, the initial values of the model parameters $\hat{A}_{1} \hat{A}_{0}$ and $\hat{B}_{0}$ can be selected as $0.01,0.02$ and 0.015 , respectively, such that $|\operatorname{det}(\bar{S}(\theta(0)))|>\rho$.

Let the dominant closed-loop poles be placed with a bandwidth of roughly $10 \mathrm{rad} / \mathrm{s}$ and with a damping factor of 0.75 , and this characteristic polynomial be given by

$$
\Xi(p)=p^{4}+65 p^{3}+1475 p^{2}+14375 p+62500
$$

The required known a priori control parameters used for simulations are listed in Table 2 .

\section{Table 2: Design parameters}

\begin{tabular}{ll}
\hline Parameter & Value \\
\hline$\phi(0)$ & 0 \\
$c_{1}$ & 0.1 \\
$\beta_{1}$ & 15 \\
$\beta_{2}$ & 10 \\
$\alpha$ & 1 \\
$k$ & 12.3 \\
$\mu$ & 1.1 \\
$d(0)$ & 1 \\
$\gamma(t)$ & 1 \\
\hline
\end{tabular}

(ii) Step 2: Update $\theta(t)=\left[\begin{array}{lll}\hat{A_{1}} & \hat{A_{0}} & \hat{B}_{0}\end{array}\right]^{T}$ using the update law eqn. 18.

(iii) Step 3: By the certainty equivalence principle, the control parameters $R_{1}\left(\theta_{a}(t)\right), \quad R_{0}\left(\theta_{a}(t)\right), \quad S_{1}\left(\theta_{a}(t)\right)$ and 
$S_{0}\left(\theta_{a}(t)\right)$ were calculated from the following pole placement technique:

$$
\begin{aligned}
\left(p^{2}+\hat{A}_{1} p+\hat{A}_{0}\right) & \left(p^{2}+R_{1}\left(\theta_{a}(t)\right) p+R_{0}\left(\theta_{a}(t)\right)\right) \\
+ & \hat{B}_{0}\left(S_{1}\left(\theta_{a}(t)\right) p+S_{0}\left(\theta_{a}(t)\right)\right)=\Xi(p)
\end{aligned}
$$

To track a step input, $T_{0}$ was given by following:

$$
T_{0}=\frac{62500}{\hat{B}_{0}}
$$

(iv) Step 4: The implementation of the control law to track a reference step input can be given by

$$
\begin{aligned}
p^{2} u_{f}(t)= & -R_{1}\left(\theta_{a}(t)\right) p u_{f}(t)-R_{0}\left(\theta_{a}(t)\right) u_{f}(t)+T_{0} r_{f}(t) \\
& -S_{1}\left(\theta_{a}(t)\right) p y_{f}(t)-S_{0}\left(\theta_{a}(t)\right) y_{f}(t)+n_{0}(t) \\
\bar{u}(t)= & p^{2} u_{f}(t)+\lambda_{1} p u_{f}(t)+\lambda_{0} u_{f}(t) \\
u(t)= & \bar{u}(t)+\frac{10}{p} \bar{u}(t)
\end{aligned}
$$

where $\lambda_{1}=50$ and $\lambda_{0}=625$ as given in eqn. 38 .

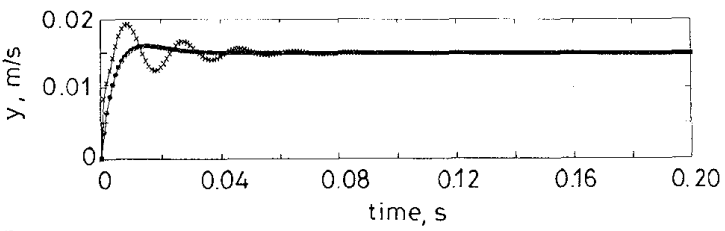

Fig.3 Trajectories of control system subject to $C_{d}=0$ : velocity output $y(t)$

$x-x-x$ suboptimal control scheme

O-O-O AMFC

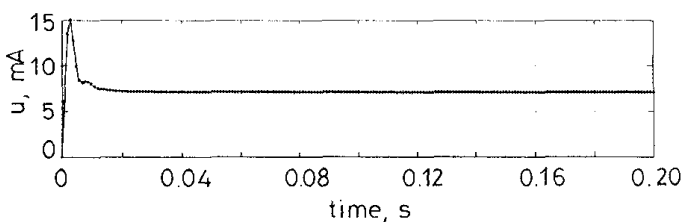

Fig.4 Trajectories of control system subject to $C_{d t}=0$ : actuator signal

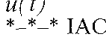

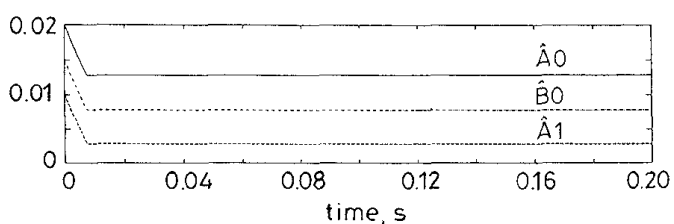
Fig.5 Trajectories of control system subject to $C_{d}=0$ : system parame-

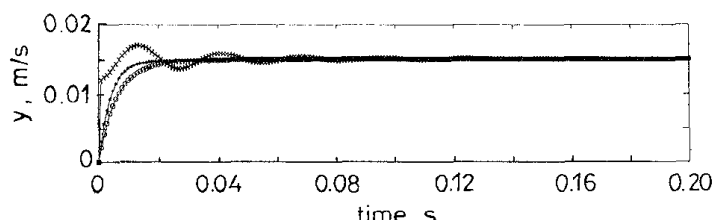

Fig. 6 Trajectories of control system subject to $C_{d}=9.8 \times 10^{6}$ : velocity output $y(t)$

$x-x-x$
$* * *$
suboptimal control scheme O-O OAMFC

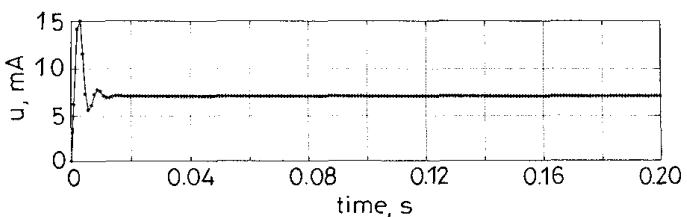

Fig.7 Trajectories of control system subject to $C_{d}=9.8 \times 10^{6}$ : actuator $\underset{* \rightarrow-* \text { IAC }}{\text { signal } u(t)}$

452

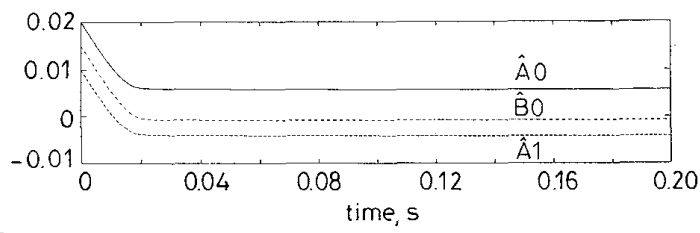

Fig.8 Trajectories of control system subject to $C_{d^{\prime}}=9.8 \times 10^{6}$ : system parameters

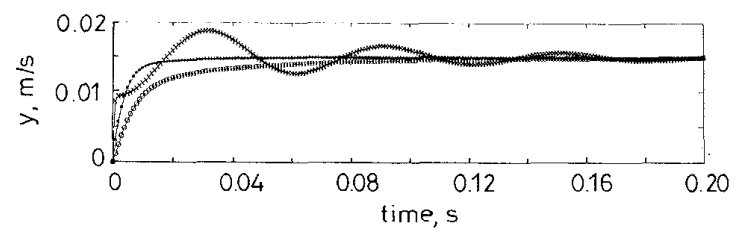

Fig.9 Trajectories of control system subject to $C_{d}=39.6 \times 10^{6}$ : velocity output $\psi(t)$

$\underset{x-x \rightarrow \text { suboptimal control scheme }}{x-* *}$

O-O-O AMFC

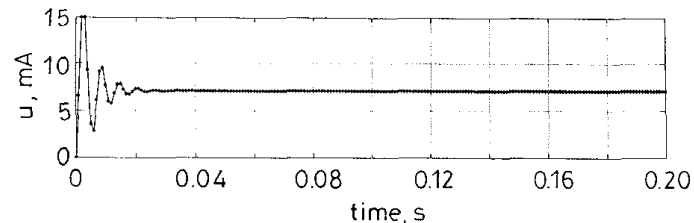

Fig. 10 Trajectories of control system subject to $C_{d}=39.6 \times 10^{5}:$ actuator signal $u$

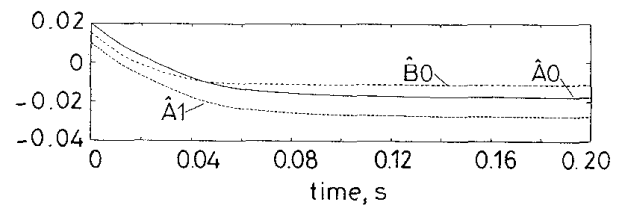

Fig. 11 Trajectories of control system subject to $C_{d}=39.6 \times 10^{6}:$ system parameters

Following the design procedure, a series of simulation studies are made, for various load disturbances, to evaluate the performance by the proposed scheme. These results are compared with those of the suboptimal PID controller [4] and that of the adaptive model following control scheme [5]. These simulations are done by a digital computer using the Simnon software package with a step size $0.001 \mathrm{~s}$. To investigate the effect of the load disturbance on the hydraulic system, $n$ is chosen to be 2 . Throughout the simulations, the reference velocity input is kept at $0.015 \mathrm{~m} / \mathrm{s}$. The performances of the system with $C_{d}=0, C_{d}=9.8 \times 10^{6}$, $C_{d}=39.2 \times 10^{6}$ and $C_{d}=9.8 \times 10^{6}+0.98 \times 10^{6} \sin (40 \pi t)$ are illustrated in Figs. 3-5, 6-8, 9-11 and 12-14, respectively. The simulation results are compared to that of the suboptimal controller which has the following form:

$$
\begin{aligned}
u(t)=k_{p}[(r(t)-y(t)) & +\frac{1}{k_{i}}\left\{\int_{0}^{t}(r(t)-y(t)) d t\right\} \\
& \left.+k_{d}\left\{\frac{d}{d t}(r(t)-y(t))\right\}\right]
\end{aligned}
$$

This controller minimises the cost function ISE (integral square error) criterion. The detailed design procedure of the suboptimal controller can be found from 
[4]. From the design procedure the parameters $k_{p}, k_{i}$ and $k_{d}$ subject to various cases of uncertainties are shown in Table 3.

Table 3: Design parameters for PID controller

\begin{tabular}{llll}
\hline$k_{p}$ & $k_{i}$ & $k_{d}$ & $C_{d}$ \\
\hline 1980 & 0.032 & 0.0027 & 0 \\
1980 & 0.043 & 0.0023 & $9.8 \times 10^{6}$ \\
1980 & 0.018 & 0.0022 & $39.2 \times 10^{6}$ \\
1980 & 0.043 & 0.0023 & $9.8 \times 10^{6}+0.98 \times 10^{6} \sin (40 \pi t)$ \\
\hline
\end{tabular}

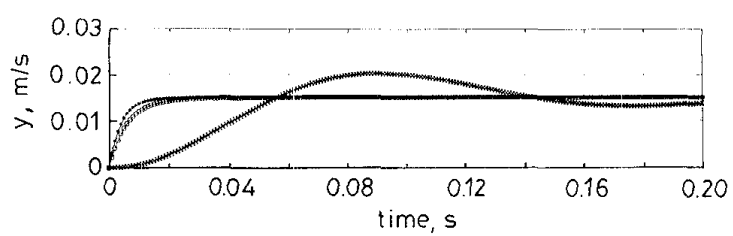

Fig. 12 Trajectories of control system subject to $C_{d}=9.8 \times 10^{6}+$ $0.98 \times 10^{6} \sin (40 \pi t)$ : velocity output $u(t)$

$x-x-x$ suboptimal control scheme

O-O-O AMFC

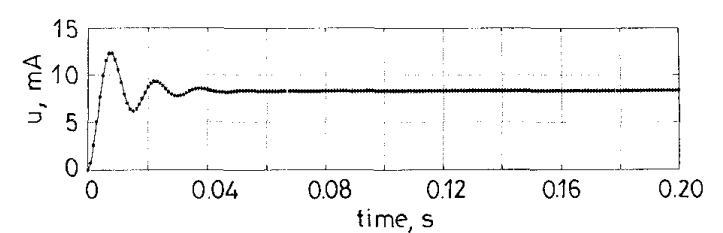

Fig. 13 Trajectories of control system subject to $C_{d}=9.8 \times 10^{6}+$ $0.98 \times 10^{6} \sin (40 \pi t)$ : actuator signal u(t)

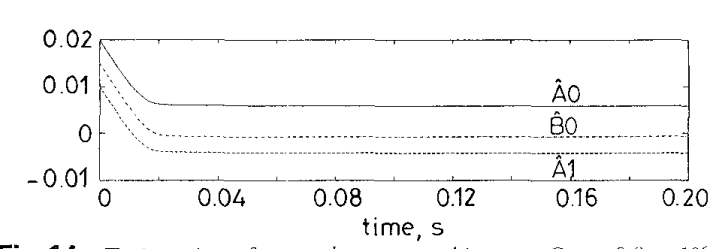

Fig. 14 Trajectories of control system subject to $C_{i t}=9.8 \times 10^{6}+$ $0.98 \times 10^{\circ} \sin (40 \pi t):$ system parameters

The simulation results are also compared to that of the adaptive model following control (AMFC) scheme [5]. In the AMFC scheme, the design parameters used for simulations are the same as that in [5] since all conditions given in eqns. 31, 32 and 41 in [5] are satisfied.

\subsection{Discussions}

The above simulations indicate that:

(i) For the case $C_{d}=0$, the velocity responses performed by the IAC and AMFC schemes shown in Figs. 3-5 are almost the same and slightly lag behind that of the suboptimal PID controller. In this case, the response performed by the IAC scheme behaves with a small amount of steady state error, about $0.3 \%$ of the desired value, and a small amount of overshoot since the damping factor of the desired closed-loop characteristics is selected as 0.75 .

(ii) As to $C_{d}=9.8 \times 10^{6}$ shown in Figs. 6-8, the responses performed by the AMFC scheme and suboptimal PID controller are observed to be inferior to that of the IAC scheme. Also, the response of the suboptimal PID controller shows large overshoot and oscillation.

(iii) From Figs. 3-11, it is also observed that the velocity responses of the AMFC scheme show slower response characteristics and that of the suboptimal controller exhibit large oscillation as the magnitude of the load disturbance increases. However, the responses of the proposed IAC scheme behave well only with a small amount of undershoot. This feature can be explained as follows. At some time, if the determinant of the Sylvester matrix $\bar{S}(\bar{\theta}(t))$ is smaller than the prespecified controllable margin $\rho$, then the estimate is replaced by the last past one which we can use to design a stabilising controller, and the exciting signal is fed back to the controlled plant simultaneously. Finally, when the excitation becomes poor again, the estimate still can be used to design the corresponding stabilising controller.

(iv) As to $C_{d}=9.8 \times 10^{6}+0.98 \times 10^{6} \sin (40 \pi t)$ shown in Fig. 6, the response of the velocity performed by the IAC scheme shows more satisfactory results for the disturbance with unknown as well as time-varying characteristics than that of the AMFC scheme and the suboptimal PID controller.

(v) From the simulation results, because there exist parametric uncertainty and disturbance, the estimated parameters obtained from estimation algorithm will not approach the true values but when used for designing the adaptive controller, the controlled system can be stabilised all the time.

(vi) From the above discussions, the proposed IAC scheme can be a promising way to tackle the problem of controlling the velocity of hydraulic servosystems subject to time-varying external velocity-dependent load disturbances.

\section{Conclusion}

In this paper, we have proposed a robust certainty equivalence based adaptive control scheme with selfexcitation capability, which is an outgrowth of the work of $[16,18,24]$, to the problem of controlling the velocity of electrohydraulic servosystems subject to unmodelled dynamics and external load disturbance. The estimator used for ideritifying the parameters of the plant model is similar to that proposed by [16-18]. It is shown that the proposed adaptive control scheme using the certainty equivalence principle and pole placement technique can guarantee the boundednesses of the input and output of the controlled plant. From the simulation results, the proposed adaptive control scheme is fairly robust to the systems with unmodelled dynamics and unknown as well as time-varying load disturbance, as compared with that of the suboptimal PID control scheme arid that of the AMFC scheme.

\section{Acknowledgment}

The authors would like to thank the reviewers for their constructive comments.

\section{References}

1 MERRITT, H.E.: 'Hydraulic control systems' (Wiley, New York, 1967)

2 PORTER, B., and TATNALL, M.L.: 'Performance characteristics of an adaptive hydraulic servo-mechanism', Int. J. Control, 1970,11 . pp $741-757$

3 KULKARNI, M.M., TRIVEDI. D.B., and CHANDRASEKHAR, J.: "An adaptive control of an electro-hydraulic position control system'. Proceedings of American Control Conference, 1984 , pp. $443-448$

4 YUN, J.S., and CHO, H.S.: 'A suboptimal design approach to the ring diameter control for ring rolling processes', Trans. ASME, J. Dyn. Syst. Meas. Control, 1985, 107, pp. 207-212

5 YUN, J.S and CHO H.S. "Adaptive model following control of electrohydraulic velocity control systems subjected to unknown electrohydraulic velocity control systems subjected to
disturbances', IEE Proc. D, 1988, 135, (2), pp. 149-156 
6 LEQUOC, S., CHENG, R.M.H., and LIMAYE, A.: 'Investigation of an electrohydraulic servovalve with tunable return pressure and drain orifice', Trans. ASME, J. Dyn. Syst. Meas. Control, 1987, 109

7 KREISSELMEIER, G.: 'An indirect adaptive controller with a self-excitation capability', IEEE Trans. Autom. Control, 1989, AC34. (5) pp. 524-528

8 KREISSELMEIER, G.: 'Adaptive observers with exponential rate of convergence', IEEE Trans. Autom. Control, 1977, AC-22, (1), pp. $2-8$

9 KREISSELMEIER, G., and SMITH, M.C.: 'Stable adaptive regulation of arbitrary nth-order plants', IEEE Trans. Autom. Control, 1986, AC-31, (4), pp. 299-305

10 GIRI, F. M'SAAD, M. DION, J-M, and DUGARD, L.: 'On the robustness of discrete-time adaptive linear controller'. Presented at 1988 Proceedings IFAC Workshop Robust Adaptive Control, Newcastle, Australia, Aug. 1988

11 GIRI, F., M'SAAD, M., DION, J.-M., and DUGARD, L.: 'On the robustness of discrete-time adaptive (linear) controllers', Automatica, 1991, 27, (1), pp. 153-159

12 ELLIOTT, H., CRISTI, R., and DAS, M.: 'Global stability of adaptive pole placemerit algorithms', IEEE Trans. Autom. Control 1985, AC-30, pp. $348-356$

13 PENG, H.-J., and CHEN, B.-S.: 'A robust indirect adaptive regulation algorithm with self-excitation capability', IEEE Trans. Autom. Control, 1993, AC-38, (2)

14 ELLIOTT, H.: 'Direct adaptive pole placement with application to nonminimum phase systems', IEEE Trans. Autom. Control, 1982, AC-27, pp $720-722$

15 ÅSTROM, K.J., and WITTENMARK, B.J.: 'Adaptive control' (Addison-Wesley Publishing Company, 1989)

16 KRAUSE, J.M., and KHARGONEKAR, P.P.: 'A comparison of classical stochastic estimator and deterministic robust estimation', IEEE Trans. Autom. Control, 1992, AC-37, (7), pp. 994 1000

17 KRAUSE, J.M., STEIN, G., and KHARGONEKAR, P.P. 'Toward practical adaptive control'. Proceedings of the American Control Conference, June 1989 , pp. 993-997

18 KRAUSE, J.M., and KHARGONEKAR, P.P.: 'Parameter identification in the presence of nonparametric dynamic uncertainty', Automatica, 1990, 26, (1), pp. 113-123

19 ÁSTROM, K.J.: 'Robustness of a design method based on assignment of poles and zeros', IEEE Trans. Autom. Control, 1980 , AC-25, (3), pp. 588-591

20 MILLER, R.K., and MICHEL, A.N.: 'Ordinary differential equations' (Academic, New York, 1982)

21 ANDERSON, B.D.O., and JOHNSTONE, R.: 'Global adaptive pole positioning', IEEE Trans. Autom. Control, 1985, AC-30, (1), pp. $11-22$

22 HAHN, W.: 'Stability of motion' (Springer-Verlag, New York, 1967)

23 VIDYASAGAR, M.: 'Nonlinear systems analysis' (Prentice-Hall, 1993)

24 MIDDLETON, R.H., and GOODWIN, G.C.: 'Digital control and estimation: A unified approach' (Prentice-Hall International Inc., 1990)

25 GOODWIN, G.C., and TEOH, E.K.: 'Persistency of excitation in the presence of possibly unbounded signals', IEEE Trans. Autom. Control, 1985, AC-30, pp. 595-597

\section{Appendix}

\subsection{Proof of Proposition 2}

From [14] and eqn. 27, we have

$\theta_{c}^{\mathrm{T}}(t) \mathbf{S}(\Theta) \mathbf{L} \phi(t)=T(p) r_{f}(t)-S\left(\theta_{a}(t), p\right) w_{f}(t)+n_{0}(t)$

where $\theta_{c}(t)=\left[\begin{array}{lllll}R_{n-1}\left(\theta_{a}(t)\right) & \ldots & R_{0}\left(\theta_{a}(t)\right) & S_{n-1}\left(\theta_{a}(t)\right) & \ldots\end{array}\right.$ $\left.S_{0}\left(\theta_{a}(t)\right)\right]^{T}$ and $L$ is a known full rank matrix. Since $\theta_{c}^{T}(t) S(\Theta) L$ is not a zero vector, we can take $v^{T}=$ $L^{T} S^{T}(\Theta) \theta_{c}(t) /\left\|L^{T} S^{T}(\Theta) \theta_{c}(t)\right\|$. Dividing both sides of eqn. 44 by $\left\|L^{T} S^{T}(\Theta) \theta_{c}(t)\right\|$ and $\mu \bar{d}(t)$ results in

$$
\begin{aligned}
& \frac{\left|\mathrm{v}^{\mathrm{T}} \phi(t)\right|}{\mu \bar{d}(t)} \geq k_{1} \frac{\left|n_{o}(t)\right|}{\mu \bar{d}(t)}-k_{1} \frac{\left|S\left(\theta_{a}(t), p\right) w_{f}(t)\right|}{\mu \bar{d}(t)}-k_{1} \frac{\left|T(p) r_{f}(t)\right|}{\mu \bar{d}(t)} \\
& 0<k_{1}<\infty
\end{aligned}
$$

Since $\left|T(p) r_{f}(t)\right| \leq n \max _{i}\left|T_{i}\right| \bar{r}$ is bounded and, from eqns. 16,27 and 28, the signal $\left|S\left(\theta_{a}(t), p\right) w_{f}(t)\right| / \mu \bar{d}(t)$ is also bounded, we have

$$
\frac{\left|\mathbf{v}^{\mathrm{T}} \phi(t)\right|}{\mu \bar{d}(t)} \geq k_{1} \kappa-\varrho(t)
$$

where $\rho(t)=\left(k_{1}\left|S\left(\theta_{a}(t), p\right) w_{f}(t)\right|+\left(\alpha-k_{1}\right) n \max _{i}\left|T_{i}\right| \bar{r}\right) /$ $(\mu \bar{d}(t))$ is bounded.

\subsection{Derivation of mathematical model}

Referring to Fig. 1 and orifice law, the load flow rate $\left(Q_{l}\right)$ of the servovalve to the actuator input current $(u)$ is given by $[1,5,6]$

$$
Q_{l}=K u \sqrt{P_{s}-\operatorname{sign}(u) P_{l}}
$$

where $K$ is the valve constant, $P_{s}$ is the supply pressure $P_{l}$ is the load pressure across the cylinder, and $\operatorname{sign}(u)=1$, for $u>0, \operatorname{sign}(u)=-1$, for $u<0$. For the application of the adaptive control, the linearised system of eqn. 47 is obtained for an arbitrary operating point in the following:

$$
Q_{l}=K_{q} u-K_{c} P_{l}
$$

where $K_{q}=K \sqrt{ }\left(P_{s}-\operatorname{sign}\left(u_{*}\right) P_{l^{*}}\right)$ is the flow gain, $K_{c}=$ $K \mid u_{*} /\left\{\sqrt{ }\left(P_{s}-\operatorname{sign}\left(u_{*}\right) P_{l^{*}}\right)\right\}$ the pressure coefficient and "*, means the nominal operating point. The continuity equation of the servovalve and the cylinder chamber is given by

$$
Q_{l}=C_{a} \dot{\psi}+C_{l} P_{l}+\left(\frac{V_{t}}{4 C_{o}}\right) \dot{P}_{l}
$$

where $C_{a}$ is the piston ram area, $C_{l}$ is the total leakage coefficient, $V_{t}$ is the total volume of the valve and the cylinder chamber, $C_{0}$ is the bulk modulus of the oil, and $\dot{\psi}$ is the velocity of the piston. To determine the minimum and maximum values of the parameters $K_{q}$ and $K_{c}$ the following physical consideration can be utilised:

$$
\frac{\left|P_{l}\right|}{P_{s}} \leq C_{p}
$$

where $C_{p}$ is an arbitrary positive number less than 1 and is chosen as $2 / 3$ for the reason that power elements are sized such that $P_{l}$ does not exceed $2 / 3 P_{s}$ for the maximum loads normally expected. Thus, the minimum and maximum values of $K_{q}$ and $K_{c}$ can be obtained as

$$
\left.\begin{array}{c}
K \sqrt{\frac{1}{3} P_{s}} \leq K_{q} \leq K \sqrt{\frac{4}{3} P_{s}} \\
\frac{K\left|u_{*}\right|}{2 \sqrt{\frac{4}{3} P_{s}}} \leq K_{c} \leq \frac{K\left|u_{*}\right|}{2 \sqrt{\frac{1}{3} P_{s}}}
\end{array}\right\}
$$

which imply that only the bounds of the magnitudes of $K_{q}$ and $K_{c}$ are known. It is assumed that the magnitudes of $K_{a}$ and $K_{c}$ are slowly time varying within the bounds. Under the assumption that Coulomb friction between the piston and sleeve is negligible, the equation of motion of the piston is given by

$$
C_{a} P_{l}=m \ddot{\psi}(t)+C_{\nu} \dot{\psi}(t)+F_{l}(\dot{\psi}(t))
$$

where $m$ is the total mass of piston and load, and $C_{p}$, is the viscous damping coefficient. It is assumed that the external load disturbance, $F_{f}(\psi(t))$, is differentiable with $\psi(t)$ and is frequently given by

$$
F_{l}(\dot{\psi}(t))=C_{d} \dot{\psi}^{n}(t)
$$

where $C_{d}=\delta F_{l} /\left.\delta \psi(t)\right|_{\psi} *(t)$ and $n$ is a positive integer number. The above equation indicates that the load disturbance depends on the velocity of the piston, which acts as a nonlinear damper whose damping coefficient varies with the velocity [5]. Hence, letting $y(t)=$ $\psi(t)$, we obtain the following linear differential equation with parametric uncertainties and external load disturbance:

$$
\ddot{y}(t)+A_{1} \dot{y}(t)+A_{0} y(t)=B_{0} u(t)+w(t)
$$

where $C_{b}=m /\left(4 C_{o}\right), K_{e}=K_{c}+C_{l}, A_{0}=\left(C_{a}{ }^{2}+K_{e} C_{p}\right)$ $\left(C_{b} m\right), A_{1}=\left(K_{e} m+C_{b} C_{v}\right) /\left(C_{b} m\right), B_{0}=\left(C_{a} K_{q}\right) /\left(C_{b} m\right)$, $w(t)=-C_{d 1} y^{n}(t)-C_{d 2} y^{n-1}(t) j(t), \quad C_{d 1}=K_{e} C_{d} /\left(C_{b} m\right)$, $C_{d 2}=n C_{d} / m$ and $n>1$. 